Transitory devices in public spaces: an experience of collective design from the architecture studio

PALABRAS CLAVE | ESPACIO PÚBLICO | DISEÑO COLECTIVO | MICROURBANISMO | ARPUITECTURA TEMPORAL

KEYWORDS | PUBUIC SPACE | COULECTIVEDESICN | MICRO-URBANISM I TEMPORARY ARCHITECTURE

\title{
Activaciones temporales en espacios de uso público: una experiencia de diseño colectivo desde el taller de arquitectura
}

\author{
RODRIGO AGUILAR PÉREZ* · Universidad de Chile, Chile · raguilar@uchile.cl
}

Fecha de recepción: 13 septiembre 2016 · Fecha de aceptación: 04 octubre 2016

\section{MARCO GENERAL. ARQUITECTURAS FRÁGILES}

El proyecto surge como una iniciativa que intenta, a través de la docencia centrada en el taller de diseño arquitectónico de la Universidad de Chile, permitir la ejecución de una intervención de carácter temporal y con un alto grado de impacto en un espacio público con poco uso y con características de abandono. Por otra parte, el proyecto pretende acercar la labor docente a una realidad tangible, instalando en los estudiantes algunos aprendizajes relacionados a la capacidad de diseño adecuado a contextos de lugar, fomentando el trabajo colectivo, mediante la participación de los usuarios y de los propios estudiantes.

Desde esta perspectiva, el proyecto plantea la elaboración por parte de los estudiantes, de un diseño de pequeña escala en la terraza superior de las pasarelas de la Remodelación San Borja, emplazada en la Comuna de Santiago, que hoy se encuentra en condiciones de vulnerabilidad y con un apreciable deterioro de sus espacios públicos.

\section{| ABSTRACT |}

The project presented here emerges as part of an initiative carried out in the $2^{\text {nd }}$ year studio at the Faculty of Architecture of the University of Chile in 2015. Its purpose was to execute a temporary but meaningful architectural device in a rundown and semi-abandoned public space. Additionally, the project intended to bring teaching and learning into a tangible reality, confronting students with the actual place of intervention, encouraging collective work with users in the initial stages, and the participation of students themselves in the building and assembling process in the later.

With this in mind, students were asked to develop a small-scale design for the upper terrace and catwalks of the San Borja Highrise Housing Renovation, located in the City of Santiago, whose public spaces are now in a position of vulnerability and manifest deterioration.

\section{Si el devenir de la actividad proyectual de la arquitectura a través de la historia ha sido marcado por la idea de solidez, permanencia y delimitación espacial, es al cabo de las últimas décadas donde estos paradigmas se han puesto a prueba, al incorporar una mirada que pone en relevancia el concepto de tiempo dentro del campo de dominio disciplinar.}

Bajo esta perspectiva, la arquitectura podría considerarse como una experiencia espacial y temporal, que logra mutar constantemente, con una noción del tiempo radicalmente opuesta al desafío de la noción de permanencia que caracterizó el modo de proyectar clásico. Dicho de otro modo, la arquitectura habría de recoger no solo la captura del espacio, sino que también el proceso de un devenir fluido y no necesariamente indeleble.

Si consideramos lo expresado por algunos autores, podemos reafirmar lo expuesto anteriormente:

\begin{abstract}
hoy parece más claro que nunca que nuestra civilización ha abandonado la estabilidad con la que el mundo se presentó en el pasado para, por el contrario, asumir el dinamismo de todas las energías que configuran nuestro entorno. Precisamente porque en nuestra cultura contemporánea atendemos prioritariamente al cambio, a la transformación y a los procesos que el tiempo establece modificando a través de él el modo de ser de las cosas, ya no podemos pensar en recintos firmes, establecidos por materiales duraderos sino en formas fluidas, capaces de incorporar, de hacer físicamente cuerpo con lo no estable sino con lo cambiante (Solà-Morales, 2001).
\end{abstract}

Estas aportaciones conceptuales permiten dibujar un trazado para la disciplina que pone por una parte de manifiesto la necesidad de tomar en cuenta los acontecimientos no permanentes, acciones eventuales o eventos (Tschumi, 1996) y, por otra parte, supone la puesta en crisis de los atributos materiales en la formalización de dicha arquitectura.

\footnotetext{
* Arquitecto de la Universidad de Chile (1998) y Máster en Historia, Arte, Arquitectura y Ciudad por la Escuela Técnica Superior de Arquitectura de Barcelona (ETSAB) de la Universidad Politécnica de Cataluña (UPC), España (2001). Desde 1998 imparte clases en la Universidad de Chile y desde 2006 en la Universidad de Santiago de Chile, donde actualmente se desempeña como Director del Programa de Máster Integrado en Diseño Arquitectónico. Ha sido Profesor Invitado en la Universidad Central y la Universidad de Talca.
} 
En otro ámbito discursivo, las dinámicas de la ciudad contemporánea plantean un grado de complejidad mayor en las relaciones entre sus actores y habitantes que muchas veces no alcanza a resolverse con la planificación y el diseño urbano, sobre todo en las formas temporales de la utilización del espacio de la ciudad, precisando entonces de un nuevo entendimiento $y$, por consiguiente, de nuevas estrategias para abordar ya sea usos informales, revitalización de espacios en desuso, reconversión de lugares deteriorados o bien aprovechamiento de áreas residuales, en una microescala, y muchas veces con herramientas que se encuentran en los márgenes de la normativa actual, o por lo menos, no bajo los cánones formales y establecidos de intervención en la ciudad.

En este contexto general, es posible considerar lo que hemos denominado como arquitecturas frágiles, como una respuesta a las condiciones de mutación de las condiciones de ocupación del espacio de lo público en la ciudad, y como una manera de dar forma física a un tiempo y experiencia inestables.

Antecedentes sobre intervenciones frágiles en la ciudad en el último tiempo existen de manera abundante. A nivel internacional, parece pertinente señalar la experiencia de Recetas Urbanas y más precisamente las acciones denominadas estrategias subversivas de ocupación urbana impulsadas por el arquitecto español Santiago Cirugeda. En ellas, se trata de cubrir una demanda arquitectónica de personas que no encuentran representación política, legislativa y tampoco inmobiliaria. De esta manera, Cirugeda crea y monta extensiones en espacios públicos, azoteas o modifica usos de edificios, generando prótesis urbanas que, a pesar de su carácter al borde de la legalidad y de las normativas, funcionan y solucionan problemas de vivienda y de diseño espacial, planteando por otra parte "situaciones donde se reclama el derecho innato del ciudadano a usar la ciudad en la que vive" (Cirugeda, 2011 ). Así, obras como Contenedores (Sevilla, 1997), Andamio en Scaffoldings (Sevilla, 1998)

1. Obra Castillo San Borja. Fotografía de Claudio Pizarro.

2. Andamio en Scaffoldings (1998) y Contenedores (1997), Sevilla. Santiago Cirugeda.
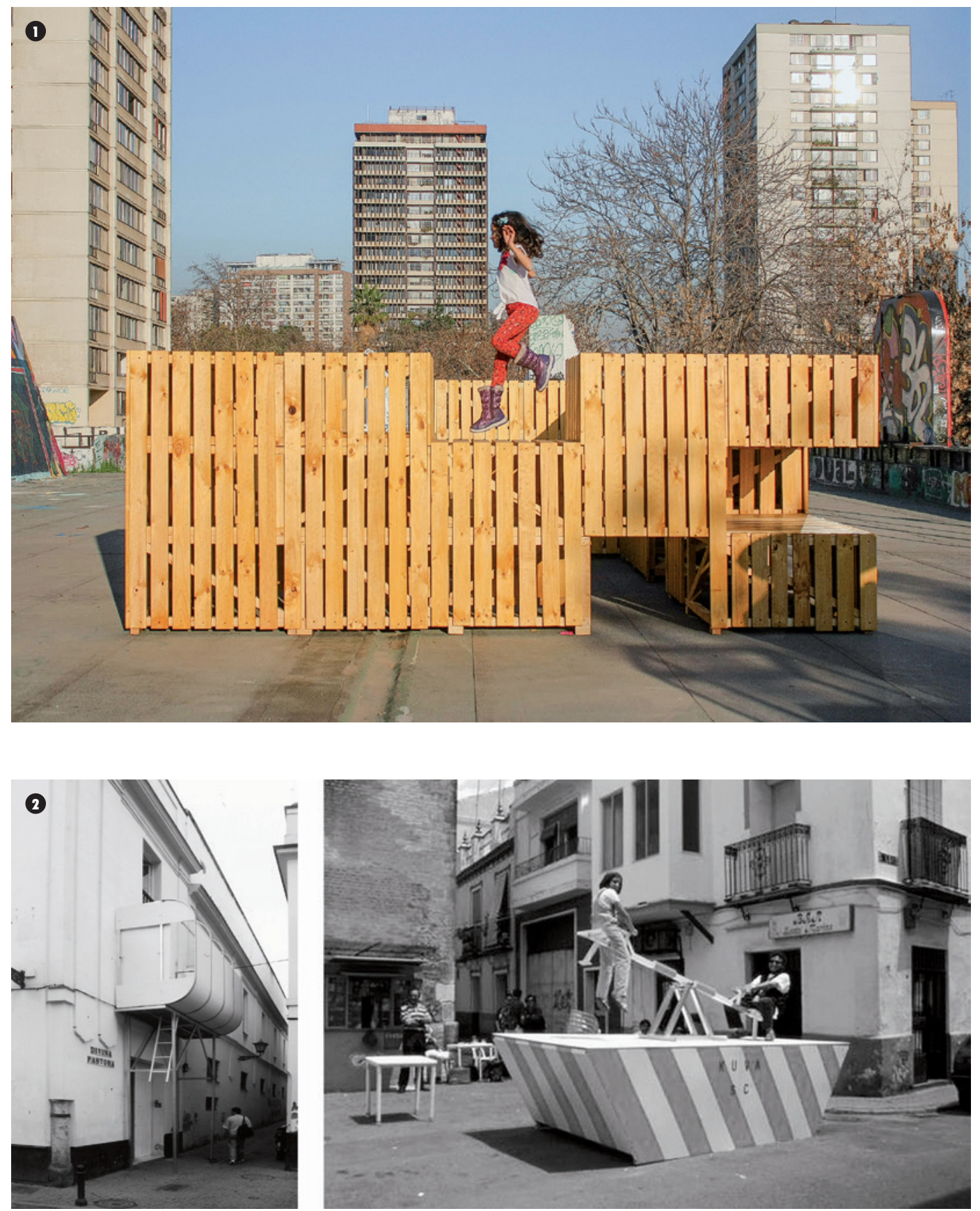

o El Niu (Girona, 2008) proponen una mirada sobre el ejercicio de la construcción de la ciudad desde una perspectiva opuesta a lo que podríamos llamar como el ejercicio formal de la arquitectura.
En el contexto de nuestro país, podemos mencionar algunos trabajos de borde, que intentan conectar los campos de la arquitectura y las artes, como Jardín Se Arrienda, realizado en el marco de la muestra Post-it City en el 
3. Imagen aerofotogramétrica de ubicación del Conjunto Habitacional San Borja y emplazamiento de la propuesta.

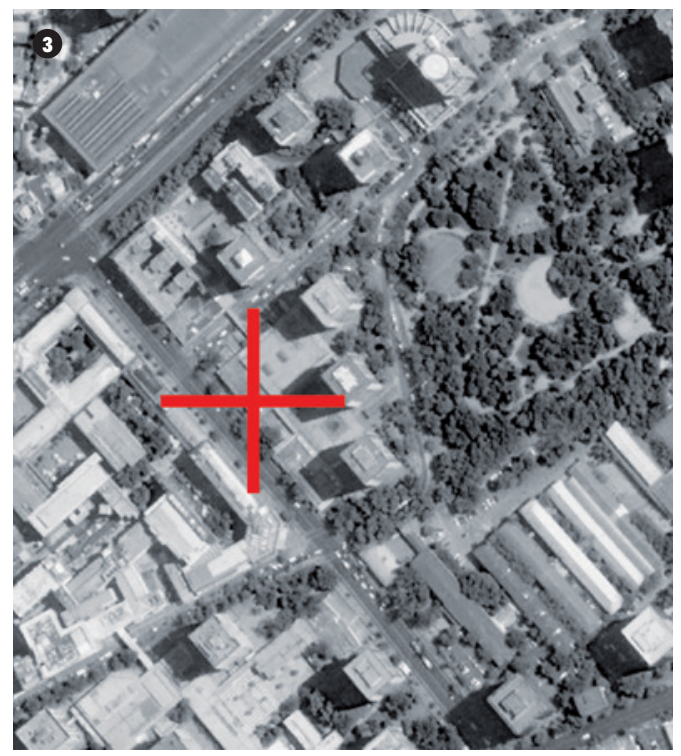

año 2009, y entendido como un proyecto colaborativo a través del contrato entre los artistas y una serie de personas para arrendar un fragmento de su jardín, con el objeto de investigar diferentes maneras de apropiación de la ciudad por parte de sus habitantes (Durán, Rubio, 2015); o bien la obra Unidad Vecinal: creatividad colectiva para el bien común, que a través de un proceso de actividades participativas en el conjunto habitacional Villa Portales, se propone la integración de las prácticas artísticas y la arquitectura, para revisitar la historia del modélico complejo urbanístico, partiendo de la pregunta sobre la posibilidad de interpretar los conflictos sociales a partir de la precarización del espacio público (Bravo, 2015).

Desde esta perspectiva, las arquitecturas frágiles, entendidas como dispositivos de pequeña escala, bajo coste, con un tiempo de duración limitado, abandonando la condición de permanencia material de largo alcance, realizadas bajo la lógica de un diseño colaborativo y en sintonía con sus habitantes, podrían ser una respuesta apropiada para activar espacios comunitarios en deterioro que la planificación formal no puede o sencillamente no alcanza a abordar.

\section{CAMPO DE ACCIÓN. PASARELAS DE SAN BORJA}

Bajo las premisas antes señaladas, el trabajo de taller, integrado por alumnos de segundo año de la carrera de arquitectura en conjunto con el equipo docente, se enfoca en el caso de estudio del conjunto habitacional San Boria, específicamente en el sistema de pasarelas sobre el nivel de la calle, cuya misión original era el establecimiento de una red de circulaciones para conectar los edificios y espacios públicos del complejo.

Emplazado en los terrenos del ex hospital del mismo nombre, el conjunto habitacional San Boria constituye un interesante ejemplo de Arquitectura Moderna y que sin embargo ha sufrido un lento pero sostenido proceso de deterioro, dado básicamente por el abandono y poco uso de sus espacios comunitarios, especialmente los que se encuentran sobre el nivel de la calle, que se han visto paulatinamente cerrados al tránsito general, básicamente y tal como argumentan los vecinos, por un problema de seguridad.

Proyectada bajo el gobierno de Eduardo Frei Montalva, en el año 1969 y construida por la Corporación de Mejoramiento Urbano desde inicios de los años setenta, el conjunto fue concebido bajo la hipótesis de repoblar y densificar el centro de la ciudad mejorando los parámetros de higiene y calidad de vida. Para ello, junto con la proyección de torres de vivienda, se concibió una serie de equipamiento comercial y de recreación, junto con una red de circulaciones separadas para automóviles y peatones, bajo la estrategia de pasarelas en un nivel separado del suelo con el objeto de conectar los edificios entre ellos y el resto de la ciudad.

Las torres fueron diseñadas por varias oficinas de arquitectura, en un esfuerzo colectivo; dentro de las que se cuentan las lideradas por Bresciani, Huidobro y Castillo Velasco (Pérez, 2006); y por Bolton, Larraín, Prieto y Lorca. Cada oficina se hizo cargo de un grupo de edificios que varía en número; sin embargo y como una forma de asegurar la uniformidad del conjunto, todos debieron considerar seis departamentos por piso con un núcleo de ascensores en plantas cuadradas, con un distanciamiento entre ellas que aseguraban condiciones de asoleamiento y ventilación, manteniendo un estándar apropiado de densidad urbana.

Aunque el conjunto quedó inconcluso de acuerdo a la planificación original, actualmente, este cuenta con 21 torres en las que viven aproximadamente diez mil personas, con su sistema propio de abastecimiento de agua potable y calefacción, además de locales comerciales, las pasarelas antes mencionadas y un parque adyacente.

En el último tiempo hemos asistido a una polémica urbana por la posible construcción de un gimnasio en el área superior de uno de los espacios destinados a comercio, producto de una confusa normativa de copropiedad y que ha causado la preocupación de los vecinos por la supuesta privatización del espacio comunitario. Sin embargo, se han desarrollado también algunas iniciativas con mayor aceptación en la comunidad, como el caso de "Pasarelas Verdes" (Mora, 2015), bajo los lineamientos de lo que algunos autores han definido como urbanismo táctico (Freire, 2009), y que para el caso particular de este proyecto, ha intentado activar el circuito mediante el establecimiento de una serie de eventos efímeros con el objeto de rescatar el lugar para los vecinos y los habitantes de su entorno inmediato. En este sentido y aprovechando el potencial de dicho espacio, "Pasarelas Verdes" ha pretendido llevar a cabo iniciativas de bajo costo y alto impacto, como jornadas musicales, establecimiento de un huerto comunitario e incorporación de mobiliario urbano, entre otras; validadas mediante la aprobación de la comunidad, para el uso y disfrute de esta porción de ciudad hasta el momento, un tanto olvidada.

En este contexto, el proyecto desarrollado en taller propone el diseño y construcción de una estructura modular con el objeto de 
4. Planimetría general del proyecto Castillo San Borja.

5. Secciones del proyecto Castillo San Boria.

6. Proceso de montaje y construcción del dispositivo. Fotografías de Rodrigo Aguilar y Claudio Pizarro.

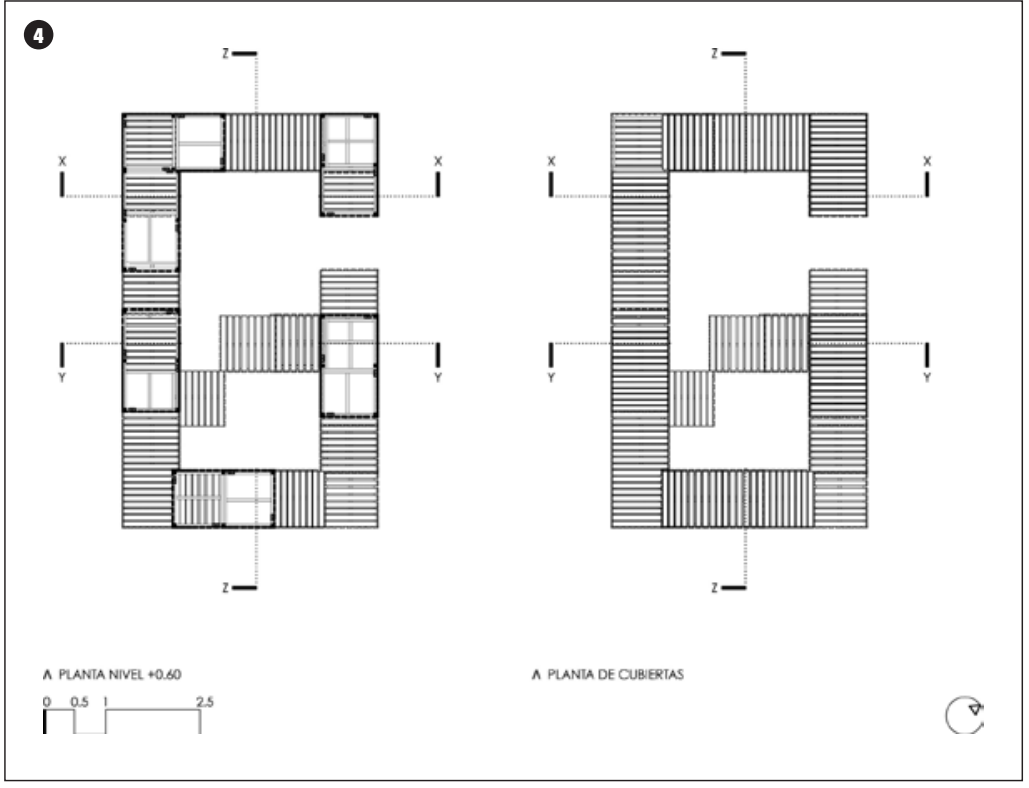

(6)
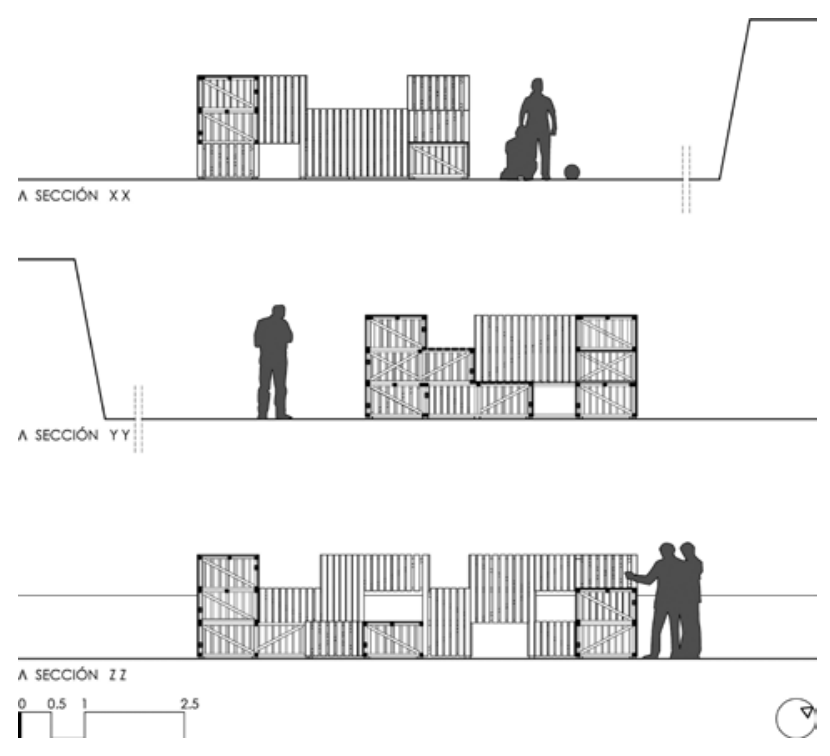

\section{(}
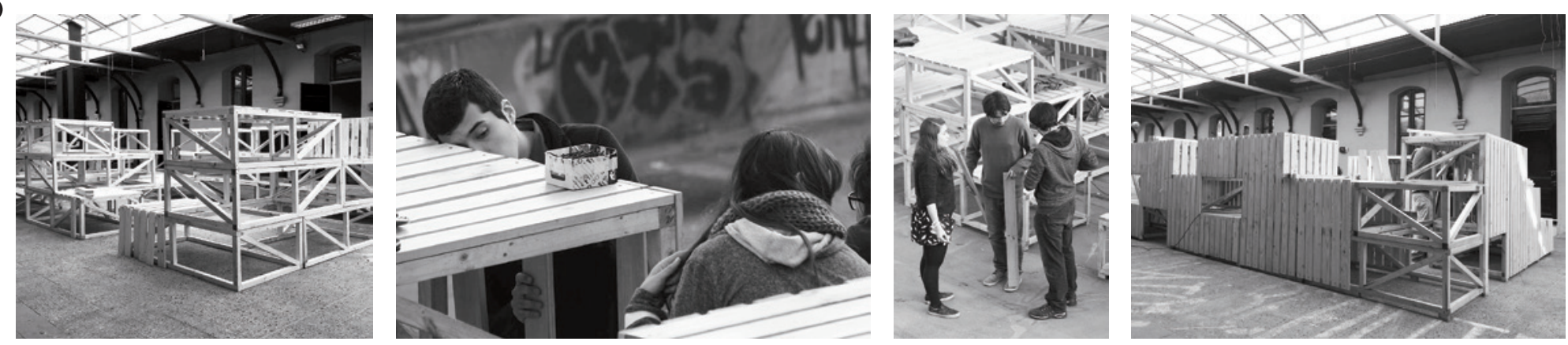

complementar las incipientes actividades de activación de las pasarelas e incentivar su ocupación activa por parte de la comunidad a partir de usos pertinentes a ello.

\section{ASPECTOS METODOLÓGICOS ACERCA DE LA EXPERIENCIA DEL DISEÑO COLECTIVO}

El proyecto se plantea como una experiencia de síntesis y acción concreta en la trayectoria del taller, luego de una primera mirada al marco teórico sobre las posibilidades de uso y activación temporal del espacio público y una segunda instancia de ejercitación de un proyecto de diseño asociado a la temática antes expuesta.

A partir de cuatro etapas secuenciales que se desarrollan en un arco temporal de diez semanas, se abarca en primer término un trabajo de exploración sobre el lugar y un diagnóstico sobre las problemáticas inherentes al contexto específico; una segunda etapa que bajo la modalidad de un concurso de ideas, pretende elaborar una variedad de respuestas posibles para el diseño de un espacio de carácter recreativo dentro del barrio seleccionado y una socialización con la comunidad sobre dichos anteproyectos; una tercera etapa de desarrollo colectivo del diseño a ejecutar en cuanto a sus detalles; $y$, finalmente, un trabajo mancomunado de ejecución constructiva.

De esta manera, la primera etapa contempla un acercamiento al lugar, a partir de visitas al conjunto, entrevistas con actores clave, reconocimiento de espacios y actividades, análisis perceptual y morfológico, diagnóstico de necesidades del barrio y definición de problemáticas. 
Dentro de esta fase, se incluyó además una encuesta a los vecinos del barrio, con la intención de recabar información sobre las necesidades de uso y las aspiraciones de los habitantes del lugar en relación a potenciales actividades que podrían implementarse para el mejor aprovechamiento del espacio urbano.

Con respecto a los resultados de la encuesta, cabe mencionar que de las 186 respuestas obtenidas, el 63,44\% manifestó un grado de pertenencia con respecto a las pasarelas y terrazas, así como el 83,20\% manifestó una valoración positiva de estos espacios para la comunidad. Por otra parte, dentro de las respuestas sobre las prioridades de programa para las pasarelas, los encuestados se manifestaron mayoritariamente por actividades recreativas familiares, espacios para deporte y recreación, juegos para niños y eventos culturales. Con esta información, fue posible por una parte derribar la percepción inicial sobre la indiferencia que el lugar podría generar en los vecinos, y por otra, establecer un orden de prioridades para los posibles y potenciales usos que podrían albergar las pasarelas con aceptación dentro de la comunidad.

Con el material recolectado, la segunda etapa incluye la formulación de ideas de diseño, elaboración de estrategias e imágenes objetivos, además de la formulación de una planimetría básica para comprender el alcance de los trabajos. Esta etapa incluyó además una jornada de socialización de los trabajos realizados a partir de una presentación a la comunidad, y la elección del anteproyecto más adecuado para su ejecución, de acuerdo a parámetros de coherencia con las problemáticas detectadas, pertinencia de la propuesta general, adecuación del diseño al contexto y a las actividades que se proyectan y finalmente a la economía de medios para llevar a cabo la solución ofrecida.

La tercera etapa consideró la adecuación del diseño seleccionado para su ejecución, resolución y revisión de planimetría general a partir de un trabajo colectivo del taller, desarrollo de detalles de arquitectura y

7. Modelo de la propuesta.

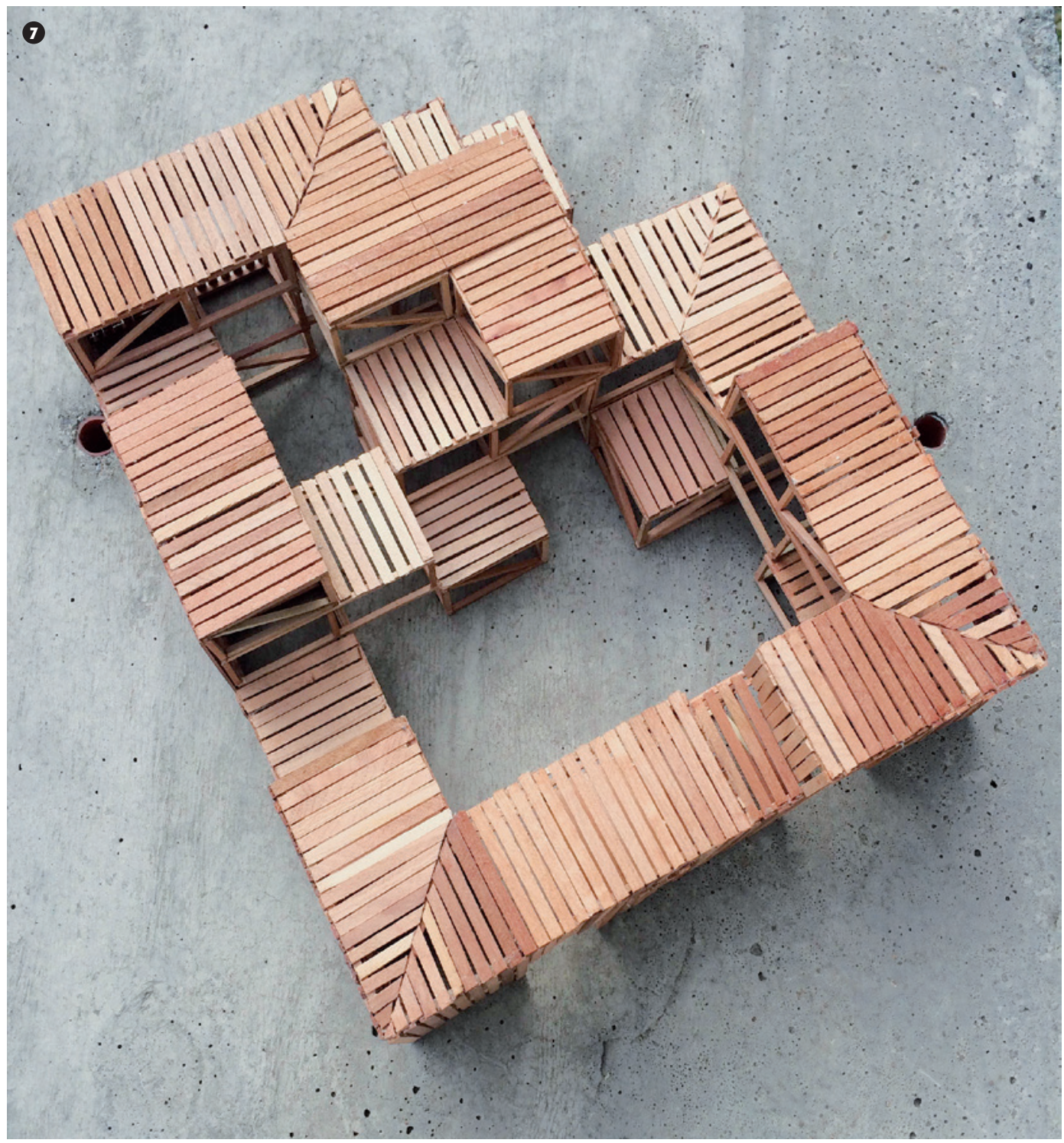

presupuesto estimado de las obras, además de la presentación del proyecto terminado a la asociación de vecinos del barrio.

Finalmente, las labores enunciadas del trabajo seleccionado para su ejecución en la etapa final incluyeron la organización y planificación de la construcción, compra de materiales, trabajo en terreno, ejecución de elementos en taller, montaje en obra y entrega del proyecto a la comunidad.

\section{RESULTADOS. \\ CASTILLO SAN BORJA}

De los anteproyectos realizados, cuyas temáticas abordaron espacios de lectura, estaciones de ejercicios, implementaciones de actividades temporales como cine al aire libre, y artefactos para juegos infantiles, finalmente se decidió desarrollar el trabajo denominado Castillo San Boria, en consideración a cuatro parámetros clave: pertinencia de la idea en relación al 
8, 9, 10 y 11. Obra Castillo San Boria. Fotografía de Claudio Pizarro.
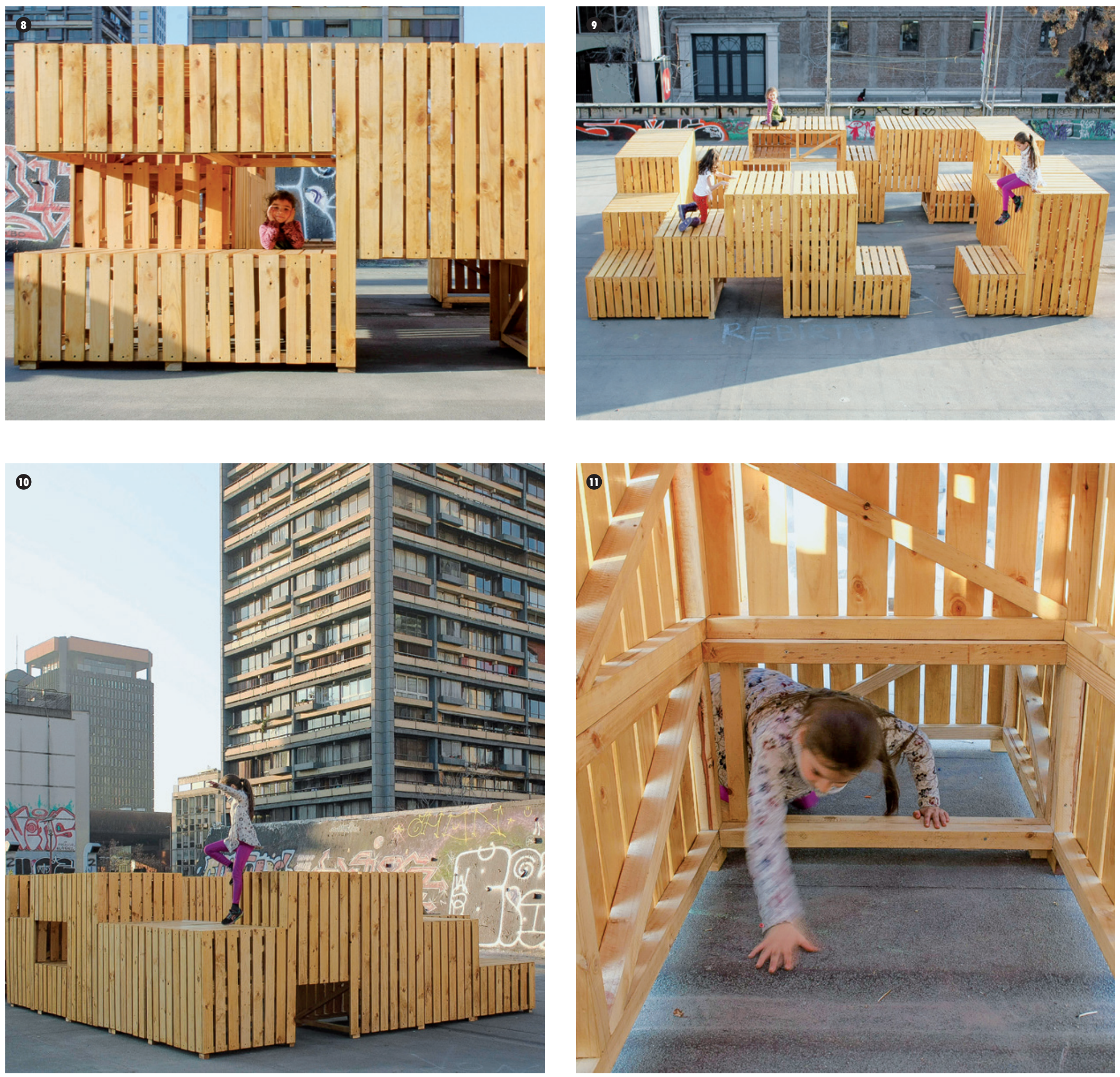
12. Obra Castillo San Boria. Fotografía de Claudio Pizarro.

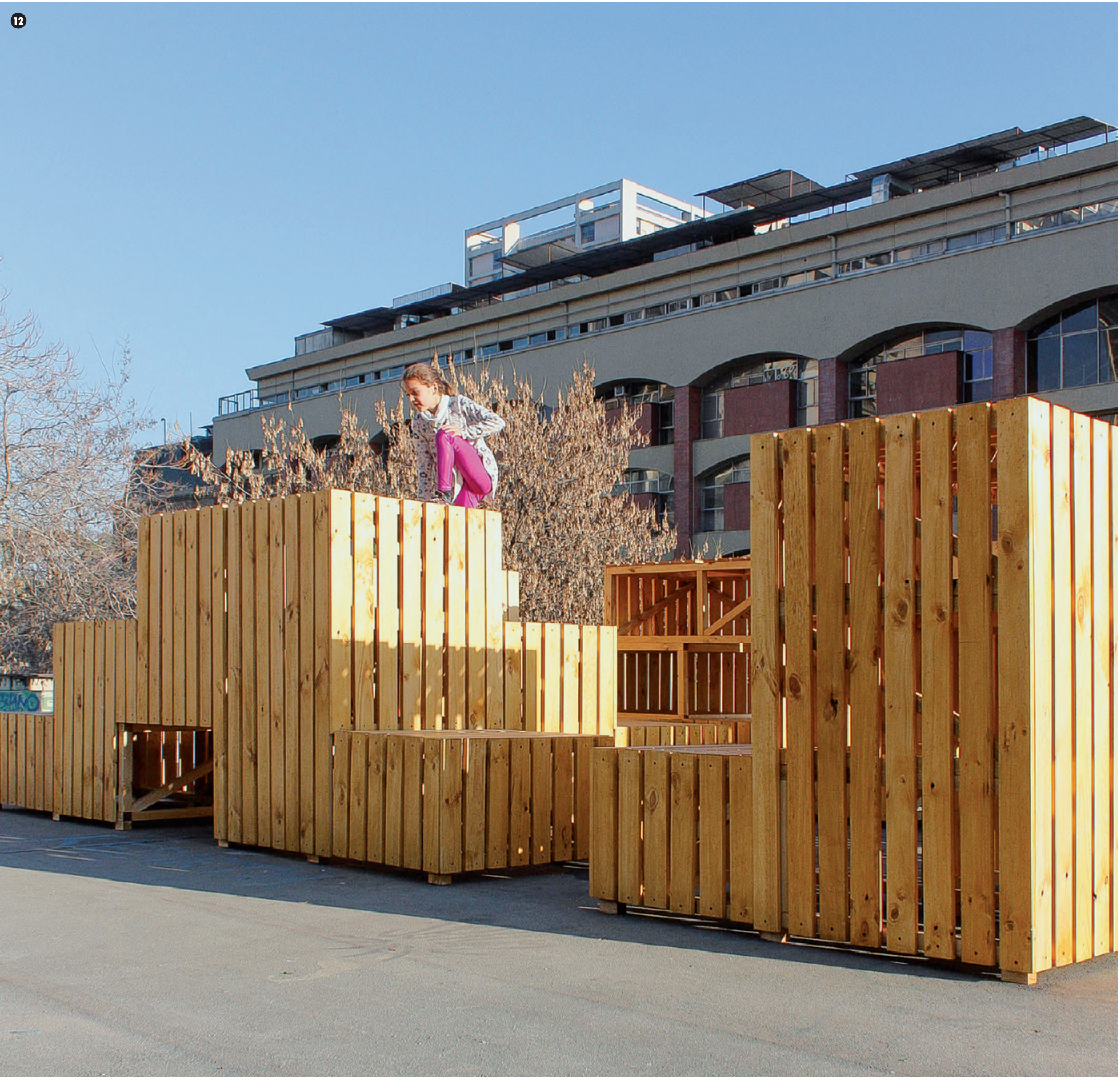


lugar de intervención, impacto de la propuesta en relación a las actividades planteadas como contribución a la activación del barrio, nivel de desarrollo del anteproyecto y finalmente, factibilidad de la propuesta, considerando su facilidad de montaje, su economía material y su rapidez constructiva.

La propuesta seleccionada proponía un dispositivo para la recreación de niños bajo el postulado de que esta población etárea constituía un sector postergado en cuanto a la ocupación de los espacios del conjunto habitacional, y que podía además congregar a los padres, abuelos o familiares de dichos niños, transformando el módulo de juegos en un punto de reunión para los habitantes. Los lineamientos de diseño del dispositivo incluyeron además la posibilidad de que el módulo pudiese albergar eventualmente una feria de productos que se realiza varias veces al año organizada por la agrupación de vecinos.

Conviene aquí precisar que el proyecto Castillo San Borja toma como referencia las intervenciones de Aldo van Eyck, y su trabajo realizado desde fines de los años 40 en el departamento de trabajos públicos de la autoridad local de Amsterdam, referido a la construcción de una serie de patios de juego público, muchos de ellos instalados en terrenos vacíos, residuales o bien en espacios destruidos como consecuencia de los bombardeos de la Segunda Guerra Mundial. En este sentido, al igual que los trabajos de van Eyck, el proyecto desarrollado busca acercarse a la hipótesis de que "el playground es, sin duda, un espacio significativo para la conformación de la sociedad y su potencial transformador es único" (Stutzin, 2015), contribuyendo por otra parte a solidificar el tejido urbano y a reforzar los lazos comunitarios, teniendo como escenario los espacios de la ciudad que se encuentran a disposición.
Materialmente, el proyecto se plantea en base a un módulo de estructura de madera de $50 \times 88 \times 160 \mathrm{~cm}$ que se dispone bajo una lógica de agregación tridimensional para configurar un volumen que se cierra parcialmente a través de una piel de listones de madera de pino y que genera dos ámbitos de ocupación: por una parte, un laberinto interior para la exploración de los niños, y por otra, un lugar de descanso en su superficie con un circuito de recorridos para saltos y juegos motrices, y que a la vez actúa como soporte de exhibición de productos para las feria de productos en tiempos ocasionales.

Las dimensiones generales del proyecto permiten el desplazamiento del usuario en su interior, como también un uso como mobiliario urbano, integrándose además a las actividades que eventualmente realiza la iniciativa "Pasarelas Verdes". De esta forma, gracias a su altura intermedia, un espacio semicontenido y un revestimiento semipermeable, el dispositivo está pensado para permitir la apropiación del espacio en diversas situaciones.

Por otra parte, las dimensiones del módulo y la combinatoria de tamaños de las piezas, permiten un óptimo aprovechamiento del material, generando mínimos excedentes.

Desde otra perspectiva, cabe agregar que en el transcurso del proyecto y luego de su ejecución, fue posible abordar y cumplir dos objetivos básicos planteados al inicio del trabajo. En una primera instancia, fue posible discutir y reflexionar sobre los aportes de la disciplina a la construcción del espacio de lo público en base a acciones detonantes de escala mínima, cuyo fin es contribuir al mejoramiento del espacio urbano y, por consiguiente, incidir directamente en las condiciones de vida de una comunidad específica. En una segunda lectura, fue posible establecer una experiencia de aprendizaje ligada a la resolución de un problema real, permitiendo la comprensión en torno al diseño en un contexto social determinado, actuando de manera colaborativa con los propios usuarios y de forma mancomunada al interior del taller.

Resulta entonces interesante poner en relevancia el valor de esta experiencia docente como un hecho concreto vinculado a la praxis en una escala acotada pero en la que convergen múltiples factores. En otras palabras, si consideramos la práctica del diseño como un ejercicio de síntesis en que la respuesta constituye la depuración de una serie de cuestiones de índole formal, material y social, el ejercicio en cuestión constituye un ejemplo de ello, añadiendo la posibilidad de tratar esta respuesta con detalle y precisión técnica.

Finalmente, sería interesante evaluar en el transcurso del tiempo el impacto de ejercicios de esta naturaleza en la comunidad, y la contribución que es posible realizar desde las prácticas pedagógicas a barrios con necesidades de espacio público insatisfechas, desde la perspectiva de lo se ha denominado como operaciones de microurbanismo.

\section{FICHA TÉCNICA}

Nombre de la obra: Castillo San Borja Localización: Conjunto Habitacional San Borja, Santiago de Chile

Autores:

Dirección del Proyecto: Rodrigo Aguilar

Pérez

Ayudante: Felipe Donoso

Monitor: Claudio Pizarro

Estudiantes de Segundo Año de la Carrera de Arquitectura: Miguel Acuña, Consuelo Albornoz, Josefa Aliaga, Francisca Álvarez, María José Ampuero, Javiera Castillo, Catalina Chaparro, Chong-A Cheong, Geraldine Díaz, Teresita Drago, Dahianet 
Inostroza, Javier Jipoulou, Marla Leiva, Diego Pino, Javier Riquelme, Diego Rozas, Marcelo San Martín, Paula Sánchez, Santiago Sierra, Tomás Silva, Sarah Tang, Francisca Vergara, Francisco Vidal, Constanza Zamora

Año de proyecto: 2016

Año de construcción: 2016

Superficie construída: 24,00 m²

Materialidad: madera de pino insigne

Fotografías: Claudio Pizarro

\section{REFERENCIAS BIBLIOGRÁFICAS}

Bravo, V. (2015). Unidad vecinal: creatividad colectiva para el bien común. En Szmulewicz, I. (ed.) Arte, ciudad y esfera pública en Chile. Santiago de Chile: Ediciones Metales Pesados.
Cirugeda, S. (2011). Reusando la ciudad, ocupando esqueletos urbanos, creando redes. Revista de Arquitectura, 17(24), 41-55, http://dx.doi. org/10.5354/0719-5427.2011.26912

Durán, A. y Rubio, L. (2015). Jardín se arrienda. En Szmulewicz, I. (ed.) Arte, ciudad y esfera pública en Chile. Santiago de Chile: Ediciones Metales Pesados.

Freire, J. (2009). Urbanismo emergente: ciudad, tecnología e innovación social. En Paisajes Domésticos, Vol. 4. Redes de Borde (18-27). España: Editorial SEPES, Entidad Estatal de Suelo.

Mora, P. (marzo, 2015). La revancha del movimiento moderno. El caso de Pasarelas Verdes. Recuperado de http://www. plataformaarquitectura.cl/cl/763980/larevancha-del-movimiento-moderno-el-caso-depasarelas-verdes
Pérez, F. (2006). Bresciani, Valdés, Castillo, Huidobro. Santiago de Chile: Ediciones ARQ.

Solà-Morales, I. (2001) Arquitectura Líquida. DC Papers, (5-6), 24-33.

Stutzin, N. (2015). Políticas del playground: los espacios de juego de Robert Moses y Aldo van Eyck. $A R Q,(91), 32-39$.

Tschumi, B. (1996). Architecture and Limits II. En Theorizing a new agenda for architecture. An anthology of architectural theory 1965-1995. New York: Princeton Architectural Press.

Tschumi, B. (1996). Architecture and disjunction. Cambridge, Massachusetts: The MIT Press. 